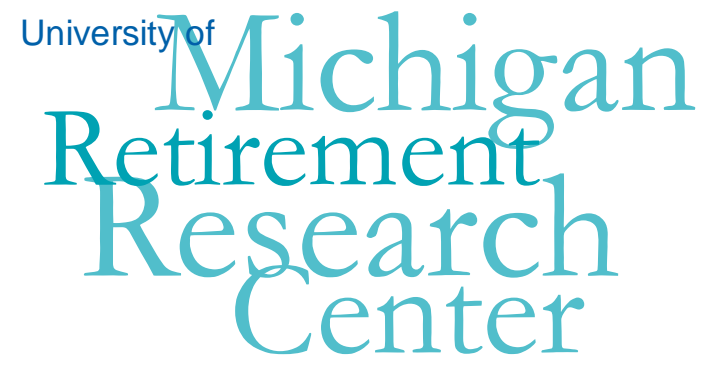

Working Paper

WP 2010-236

\title{
The Effects of Medicaid and Medicare Reforms on the Elderly's Savings and Medical Expenditures
}

\author{
Mariacristina De Nardi, Eric French and John Bailey Jones
}

\begin{tabular}{|l|l|}
\hline $\mathrm{M}$ & $\mathrm{R}$ \\
\hline $\mathrm{R}$ & $\mathrm{C}$ \\
\hline
\end{tabular}

Project \#: UM10-16 


\title{
The Effects of Medicaid and Medicare Reforms on the Elderly's Savings and Medical Expenditures
}

\author{
Mariacristina De Nardi \\ Federal Reserve Bank of Chicago and NBER \\ Eric French \\ Federal Reserve Bank of Chicago \\ John Bailey Jones \\ University at Albany, SUNY
}

October 2010
Michigan Retirement Research Center
University of Michigan
P.O. Box 1248
Ann Arbor, MI 48104
http://www.mrrc.isr.umich.edu/
(734) 615-0422

\section{Acknowledgements}

This work was supported by a grant from the Social Security Administration through the Michigan Retirement Research Center (Grant \# 10-M-98362-5-01). The findings and conclusions expressed are solely those of the author and do not represent the views of the Social Security Administration, any agency of the Federal government, or the Michigan Retirement Research Center.

\section{Regents of the University of Michigan}

Julia Donovan Darrow, Ann Arbor; Laurence B. Deitch, Bingham Farms; Denise Ilitch, Bingham Farms; Olivia P. Maynard, Goodrich; Andrea Fischer Newman, Ann Arbor; Andrew C. Richner, Grosse Pointe Park; S. Martin Taylor, Gross Pointe Farms; Katherine E. White, Ann Arbor; Mary Sue Coleman, ex officio 


\title{
The Effects of Medicaid and Medicare Reforms on the Elderly's Savings and Medical Expenditures
}

\begin{abstract}
We study a model in which retired single people optimally choose consumption, medical spending and saving while facing uncertainty about their health, lifespan and medical needs. This uncertainty is partially offset by insurance provided by the government and private institutions. We first show how well the model matches important features of the data and we analyze the degree of insurance provided by current programs. We then analyze the effects of some reforms, meant to capture changes in Medicaid and Medicare, on savings and medical expenditures.
\end{abstract}

\section{Authors’ Acknowledgements}

Mariacristina De Nardi: Federal Reserve Bank of Chicago and NBER. Eric French: Federal Reserve Bank of Chicago. John Bailey Jones: University at Albany, SUNY. De Nardi gratefully acknowledges financial support from NSF grant SES-0317872. Jones gratefully acknowledges financial support from the Social Security Administration through the Michigan Retirement Research Center (MRRC grant UM10-16). The views expressed in this paper are those of the authors and not necessarily those of the Federal Reserve Bank of Chicago, the Federal Reserve System, the National Science Foundation, the Social Security Administration or the MRRC. 


\section{Introduction}

Many poorer Americans receive health insurance through Medicaid, a public program run jointly by the federal and state governments. In 2008, total Medicaid outlays in the U.S. totalled 339 billion dollars, 2\% of GDP. An important group of Medicaid beneficiaries is the elderly and the disabled, including those with long-term care needs. The other main public health insurance program for the elderly and disabled, Medicare, reimburses only a limited amount of long-term care costs, and most elderly people do not have private long-term care insurance. As a result, Medicaid covers almost all nursing home costs of poor old recipients. In 2008, 24\% of Medicaid expenditures went to the elderly and $42 \%$ went to the disabled (some of whom are also elderly). Medicaid now assists $70 \%$ of nursing home residents. ${ }^{1}$

Because the need to pay for health care at very old ages is potentially a significant savings motive, the insurance provided by Medicaid may have significant effects on saving. Hubbard et al. [13] and Scholz et al. [18] argue that means-tested social insurance programs (in the form of a minimum consumption floor) provide strong incentives for low-income individuals not to save. Brown and Finkelstein [2] conclude that Medicaid could explain the lack of private long-term care insurance for about two-thirds of the wealth distribution. De Nardi, French and Jones [6] find that reducing the generosity of social insurance significantly increases the saving of elderly singles. Kopecky and Koreshkova [15] find that old-age medical expenses, and the coverage of these expenses provided by Medicaid, have large effects on aggregate capital accumulation.

In this paper, we study a model in which retired single people optimally choose consumption, medical spending and saving while facing uncertainty about their health, lifespan and medical needs. This uncertainty is partially offset by insurance provided by the government and private institutions. We first show how well the model matches important features of the data and we analyze the degree of insurance provided by current programs. We then study some policy reforms, meant to capture recent changes in Medicaid and Medicare, analyzing the effects of these reforms on out-of-pocket and total medical expenditures, and savings.

We extend the existing literature by focusing on Medicaid, and by analyzing its insurance role for people with different wealth and income levels,

\footnotetext{
${ }^{1}$ Statistics assembled by the Kaiser Foundation [16].
} 
health, and gender. We estimate the parameters of our model rather than calibrating them to previous studies, which might have features which are inconsistent with the model at hand. We require our model to fit well across the entire income distribution, rather than simply explain mean or median behavior. We model medical expenditure as an endogenous choice. This allows us to consider how Medicaid and Medicare reforms affect total medical spending. We model social insurance as providing a utility floor, rather than a fixed expenditure floor. This allows means-tested transfers to vary with medical needs in a way consistent with actual practice. ${ }^{2}$ Due to the richness and complexity of our framework, we focus on the post-retirement part of the life-cycle and adopt a partial equilibrium approach.

We find that the model matches the life-cycle profiles of assets and out-ofpocket medical spending for elderly singles in different cohorts and permanent income groups. It also generates an elasticity of total medical expenditures to co-pay changes that is close to the one estimated in the data.

We find that the current Medicaid system provides different kinds of insurance to households with different permanent income levels. Households in the lower permanent income quintiles are much more likely to receive Medicaid transfers, but the transfers that they receive are on average relatively small. Households in the higher permanent income quintiles are much less likely to receive any Medicaid pay-outs, but when they do, these pay-outs are very big and correspond to severe and expensive medical conditions. Therefore, Medicaid is an effective insurance device for the poorest, but also offers very valuable insurance to the rich by insuring them against catastrophic medical conditions. For this reason, we find that making Medicaid more generous reduces the elderly's savings at all permanent income levels, including the highest. Making Medicaid more generous also increases total medical expenditures, especially for lower-income households, but it decreases the out-of-pocket costs actually paid by households.

We also study the effects of changing the generosity of Medicare by reducing the co-pays that elderly people incur when consuming medical goods and services. As in the previous experiment, households become more fully

\footnotetext{
${ }^{2}$ Two recent papers contain life-cycle models where the choice of medical expenditures also affects health outcomes. In addition to having different emphases, both papers model Medicaid in ways different from our's. Feng [7] models Medicaid as an insurance policy with no premiums and extremely low - possibly zero - co-payment rates. Fonseca et al. [10] assume that the consumption floor is invariant to medical needs (private conversation with Pierre-Carl Michaud).
} 
insured by the government as a result of this reform and thus decrease their savings. This reform, however, benefits higher permanent income people more than poorer ones, because the poor were already well insured by Medicaid. Total medical expenditures rise at all ages, not only when very old, and rise proportionally more for younger people. As in the Medicaid reform, out-of-pocket medical expenditures decline.

The rest of the paper is organized as follows. In Section 2, we describe our model. In Section 3, we briefly discuss how we estimate the model's parameters. In Section 4, we describe the data used in this analysis. In Section 5 , we discuss the life cycle profiles found in the data and our estimation results. In Section 6, we perform some policy experiments. In Section 7, we conclude and discuss directions for future research.

\section{The model}

The model of consumption and medical spending decisions used in this paper comes from De Nardi et al. [6]. We focus on single people who have already retired. This allows us to abstract from labor supply and retirement decisions, and from complications arising from family dynamics such as the transition from two family members to one. We also abstract from bequest motives (see De Nardi et al. [6] for a model with a bequest motive).

We assume that people are hit by medical needs shocks, such as cancer, diabetes, a heart attack, or a broken bone. These shocks affect their marginal utility of consuming medical goods and services, and people choose how much to spend in response to these shocks. A complementary approach is that of Grossman [12], in which medical expenses represent investments in health capital, which in turn decreases mortality (e.g., Yogo [19]) or improves health. While some studies find that medical expenditures have significant effects on the health and/or survival of the elderly (Card et al. [3]; Doyle [4]), many others find small effects (Brook et al. [1]; Fisher et al. [9]; Finkelstein and McKnight [8]; Khwaja [14]); see our previous paper for a discussion. These findings suggest that the effects of medical expenditures on the health outcomes are at a minimum extremely difficult to identify. ${ }^{3}$ Given that older people have already shaped their health and lifestyle, we view our assumption

\footnotetext{
${ }^{3}$ Identification problems include reverse causality - sick people have higher health expenditures - and a lack of insurance variation - most elderly individuals receive Medicare and/or Medicaid.
} 
that their health and mortality depend on their lifetime earnings, but is exogenous to their current decisions, to be a reasonable simplification.

\subsection{The individual's problem}

Consider a single person, either male or female, seeking to maximize his or her expected lifetime utility at age $t, t=t_{r+1}, \ldots, T$, where $t_{r}$ is the retirement age. His flow utility from consumption and medical expenditures is given by

$$
u\left(c_{t}, m_{t}, \mu_{t}\right)=\frac{1}{1-\nu} c_{t}^{1-\nu}+\mu_{t} \frac{1}{1-\omega} m_{t}^{1-\omega},
$$

where $t$ is age, $c_{t}$ is consumption of non-medical goods, $m_{t}$ is total consumption of medical goods, and $\mu_{t}$ is the medical needs shifter, which affects the marginal utility of consuming medical goods and services. The consumption of both goods is expressed in dollar values. The intertemporal elasticities for the two goods, $1 / \nu$ and $1 / \omega$, can differ.

The individual faces several sources of risk, which we treat as exogenous: health status risk, survival risk, and medical needs risk. At the beginning of each period, the individual's health status and medical needs shocks are realized and need-based transfers are given. The individual then chooses consumption, medical expenditure, and saves. Finally, the survival shock hits.

Letting $h_{t}$ denote the retiree's health status, we parameterize the preference shifter for medical goods and services (the needs shock) as

$$
\begin{aligned}
\ln \left(\mu\left(h_{t}, \psi_{t}, t\right)\right)= & \alpha_{0}+\alpha_{1} t+\alpha_{2} t^{2}+\alpha_{3} t^{3}+\alpha_{4} h_{t}+\alpha_{5} h_{t} \times t \\
& +\sigma\left(h_{t}, t\right) \times \psi_{t}, \\
\sigma\left(h_{t}, t\right)^{2}= & \beta_{0}+\beta_{1} t+\beta_{2} t^{2}+\beta_{4} h_{t}+\beta_{5} h_{t} \times t \\
\psi_{t}= & \zeta_{t}+\xi_{t}, \quad \xi_{t} \sim N\left(0, \sigma_{\xi}^{2}\right), \\
\zeta_{t}= & \rho_{m} \zeta_{t-1}+\epsilon_{t}, \quad \epsilon_{t} \sim N\left(0, \sigma_{\epsilon}^{2}\right), \\
\sigma_{\xi}^{2}+\frac{\sigma_{\epsilon}^{2}}{1-\rho_{m}^{2}} \equiv & 1,
\end{aligned}
$$

where $\xi_{t}$ and $\epsilon_{t}$ are serially and mutually independent. We thus allow the need for medical services to have temporary $\left(\xi_{t}\right)$ and persistent $\left(\zeta_{t}\right)$ shocks. It is worth stressing that we do not allow any component of $\mu$ to depend on permanent income; income affects medical expenditures solely through the budget constraint. 
The individual's health status, $h_{t}$, varies between bad $\left(h_{t}=0\right)$ and good $\left(h_{t}=1\right)$. The transition probabilities for health status depend on current health, sex $(g)$, permanent income $(I)$, and age, so that the elements of the health status transition matrix are

$$
\pi_{j, k, g, I, t}=\operatorname{Pr}\left(h_{t+1}=k \mid h_{t}=j, g, I, t\right), \quad j, k \in\{0,1\} .
$$

Let $s_{t}$ denote the probability that an individual is alive at age $t+1$, conditional on being alive at age $t$. This survival probability varies with sex, health status, permanent income and age, so that $s_{t}=s_{g, h, I, t}$.

Non-asset income, $y_{t}$, is a deterministic function of sex, permanent income, and age:

$$
y_{t}=y(g, I, t)
$$

Assets evolve according to

$$
a_{t+1}=a_{t}+y_{n}\left(r a_{t}+y_{t}\right)+b_{t}-c_{t}-m_{t} q_{t} \geq 0,
$$

where $y_{n}\left(r a_{t}+y_{t}, \tau\right)$ denotes post-tax income, $y_{t}$ denotes non-asset income, $r$ denotes the risk-free, pre-tax rate of return, the vector $\tau$ describes the tax structure, $b_{t}$ denotes government transfers, and $q_{t}$ denotes the co-payment rate. We impose that if transfers are positive, the individual consumes all of his resources (by splitting them optimally between the two goods), so that $a_{t+1}=0$.

We model two important features of the health care system:

1. Private and public insurance pay the share $1-q\left(t, h_{t}\right)$ of the total medical costs incurred by the retiree. Its complement, $q\left(t, h_{t}\right)$, is the out-of-pocket share paid by the retiree.

2. Social insurance programs, such as Medicaid and SSI, provide monetary transfers that vary with financial resources and medical needs. We model these transfers as providing a flow utility floor. The transfers thus depend on the retirees' state variables, including their medical needs shocks. For a given utility floor and state vector, we find the transfer $b^{*}(\cdot)=b^{*}\left(t, a_{t}, g, h_{t}, I, \zeta_{t}, \xi_{t}\right)$ that puts each retiree's utility at the floor. Transfers then kick in to provide the minimum utility level to retirees who otherwise could not afford it:

$$
b\left(t, a_{t}, g, h_{t}, I, \zeta_{t}, \xi_{t}\right)=\max \left\{0, b^{*}\left(t, a_{t}, g, h_{t}, I, \zeta_{t}, \xi_{t}\right)\right\}
$$


Letting $\beta$ denote the discount factor, the value function for a single individual is given by

$$
\begin{aligned}
V_{t}\left(a_{t}, g, h_{t}, I, \zeta_{t}, \xi_{t}\right)= & \max _{c_{t}, m_{t}, a_{t+1}}\left\{\frac{1}{1-\nu} c_{t}^{1-\nu}+\mu\left(h_{t}, \psi_{t}, t\right) \frac{1}{1-\omega} m_{t}^{1-\omega}\right. \\
& \left.+\beta s_{g, h, I, t} E_{t}\left(V_{t+1}\left(a_{t+1}, g, h_{t+1}, I, \zeta_{t+1}, \xi_{t+1}\right)\right)\right\},
\end{aligned}
$$

subject to equations (9) and (10).

\subsection{The intratemporal allocation decision}

Suppose that at time $t$ an individual decides to spend the total $x_{t}$ on consumption and out-of-pocket payments for medical goods. If the individual faces the taste shifter $\mu_{t}$ and the co-pay rate $q_{t}$, the optimal intratemporal allocation will satisfy

$$
m_{t}=\left(\frac{\mu_{t}}{q_{t}}\right)^{1 / \omega} c_{t}^{\nu / \omega} .
$$

Equation (12) shows that the demand for medical services is less elastic than the demand for consumption goods if $\nu$ is less than $\omega$. If so, as total expenditures rise, an decreasing share will be devoted to medical goods. The relationship between income and out-of-pocket medical expenditures, however, also depends on social insurance and the distribution of the medical needs shocks.

Plugging equation (12) into the intratemporal budget constraint yields

$$
x_{t}=q_{t}\left(\frac{\mu_{t}}{q_{t}}\right)^{1 / \omega} c_{t}^{\nu / \omega}+c_{t} .
$$

Equations (12) and (13) allow us to map the utility floor $\underline{U}$ into the transfer $b_{t}$. The first step is to normalize the utility floor as a function of the consumption level $\underline{c}$ :

$$
\begin{aligned}
\underline{U} & =U(\underline{c}) \\
& \equiv \frac{1}{1-\nu} \underline{c}^{1-\nu}+\frac{1}{1-\omega} \underline{c}^{1-\omega} .
\end{aligned}
$$


Then for any realization of $\mu_{t}$ and $q_{t}$, we can solve for the expenditure floor:

$$
\begin{aligned}
\underline{x}_{t} & =x\left(\mu_{t}, q_{t} ; \underline{c}\right) \\
& =c+q_{t}\left(\frac{\mu_{t}}{q_{t}}\right)^{1 / \omega} c^{\nu / \omega}, \\
c: & \frac{1}{1-\nu} c^{1-\nu}+\mu_{t} \frac{1}{1-\omega}\left[\left(\frac{\mu_{t}}{q_{t}}\right)^{1 / \omega} c^{\nu / \omega}\right]^{1-\omega}=U(\underline{c}) .
\end{aligned}
$$

The final step is to back out the transfer itself:

$$
b\left(t, a_{t}, g, h_{t}, I, \zeta_{t}, \xi_{t}\right)=\max \left\{0, \underline{x}_{t}-a_{t}-y_{n}\left(r a_{t}+y_{t}\right)\right\} .
$$

\section{Estimation procedure}

\subsection{The method of simulated moments}

We adopt a two-step strategy to estimate the model. In the first step we estimate or calibrate those parameters that can be cleanly identified outside our model. For example, we estimate mortality rates from raw demographic data. In the second step we estimate the rest of the model's parameters with the method of simulated moments (MSM), taking as given the parameters that were estimated in the first step. In particular, we find the parameter values that minimize the difference (as measured by a GMM criterion function) between the asset and out-of-pocket medical expense profiles generated by the model and their data counterparts.

To construct the asset profiles, we sort individuals into permanent income quintiles, and 5 birth-year cohorts. The first cohort consists of individuals that were ages $72-76$ in 1996; the second cohort contains ages $77-81$; the third ages 82-86; the fourth ages 87-91; and the final cohort, for sample size reasons, contains ages 92-102. We use net worth data for 6 different years: 1996, 1998, 2000, 2002, 2004, and 2006. To construct the profiles, we calculate cell medians for all of the survivors in each year.

Regarding medical expenses, we include the following moments in our moment conditions: mean medical expenses by age and birth cohort for each half of the permanent income distribution; the 90th percentile of medical expenses in the same cells; and the first and second autocorrelations for medical expenses in each cell. Because the AHEAD medical expense data 
are reported net of any Medicaid payments, we deduct government transfers from the medical expenses generated by the model before making any comparisons. A detailed description of these moment conditions, the asymptotic distribution of the parameter estimates, the weighting matrix, and the overidentification tests, can be found in the on-line appendix for De Nardi et al. [6].

The mechanics of our MSM approach are as follows. We compute lifecycle histories for a large number of artificial individuals. Each of these individuals is endowed with a value of the state vector $\left(t, a_{t}, g, h_{t}, I\right)$ drawn from the data distribution for 1996, and each is assigned a series of health, medical expense, and mortality shocks consistent with the stochastic processes described in the model section. We give each simulated person the entire health and mortality history realized by a person in the AHEAD data with the same initial conditions. The simulated medical needs shocks $\zeta$ and $\xi$ are Monte Carlo draws from discretized versions of our estimated shock processes.

We discretize the asset grid and, using value function iteration, we solve the model numerically. This yields a set of decision rules, which, in combination with the simulated endowments and shocks, allows us to simulate each individual's net worth, medical expenditures, health, and mortality. We then compute asset and medical expense profiles from the artificial histories in the same way as we compute them from the real data.

\section{Data}

The AHEAD is part of the Health and Retirement Survey (HRS) conducted by the University of Michigan. It is a survey of individuals who were non-institutionalized and aged 70 or older in 1994. A total of 3,872 singles were interviewed for the AHEAD survey in late 1993/early 1994, which we refer to as 1994. These individuals were interviewed again in 1996, 1998, 2000, 2002, 2004, and 2006. We consider only single retired individuals in the analysis. This leaves us with 3,259 individuals, of whom 592 are men and 2,667 are women. Of these 3,259 individuals, 884 are still alive in 2006.

Our measure of net worth (or assets) is the sum of all assets less mortgages and other debts. The AHEAD has information on the value of housing and real estate, autos, liquid assets (which include money market accounts, savings accounts, T-bills, etc.), IRAs, Keoghs, stocks, the value of a farm 
or business, mutual funds, bonds, and "other" assets. We do not use 1994 assets because they were underreported (Rohwedder et al. [17]).

Non-asset income includes the value of Social Security benefits, defined benefit pension benefits, annuities, veterans benefits, welfare, and food stamps. We measure permanent income (PI) as the individual's average income over all periods during which he or she is observed. Because Social Security benefits and (for the most part) pension benefits are monotonic functions of average lifetime labor income, this provides a reasonable measure of lifetime, or permanent income.

In all waves, AHEAD respondents are asked about what medical expenses they paid out of pocket. Out-of-pocket medical expenses are the sum of what the individual spends out of pocket on insurance premia, drug costs, and costs for hospital, nursing home care, doctor visits, dental visits, and outpatient care. It includes medical expenses during the last year of life. It does not include expenses covered by insurance, either public or private. French and Jones [11] show that the medical expense data in the AHEAD line up with the aggregate statistics. For our sample, mean medical expenses are $\$ 3,712$ with a standard deviation of $\$ 13,429$ in 1998 dollars. Although this figure is large, it is not surprising, because Medicare did not cover prescription drugs for most of the sample period, requires co-pays for services, and caps the number of reimbursed nursing home and hospital nights.

After being asked about all of the components of out-of-pocket medical expenses, respondents are asked to estimate total medical expenses (out-ofpocket plus covered by Medicaid, Medicare or other insurance). Respondents in 1996 were asked to provide a dollar amount, whereas in 1998-2002 respondents were asked only to provide ranges (in 1998 the groups were less than $\$ 1,000, \$ 1,000-\$ 5,000, \$ 5,000-\$ 25,000, \$ 25,000-\$ 100,000, \$ 100,000-\$ 500,000$, or more than $\$ 500,000)$. We use the RAND imputations to infer total medical expenses from these responses. Total medical expenses average $\$ 22,094$ with a standard deviation of $\$ 74,637$. Combining this data with our measure of out-of-pocket expenses allows us to calculate co-pay rates.

In addition to constructing moment conditions, we also use the AHEAD data to construct the initial distribution of permanent income, age, sex, health, and assets that starts off our simulations. Each simulated individual is given a state vector drawn from the joint distribution of these state variables observed in 1996. 


\section{Data profiles and estimation results}

In this section, we describe the life cycle profiles of the stochastic processes that are inputs to our dynamic programming model.

\section{$5.1 \quad$ Income profiles}

We model income as a function of: a quartic in age, sex, sex interacted with age, current health status, health status interacted with age, a quadratic in the individual's permanent income ranking, and permanent income ranking interacted with age. We estimate these profiles using a fixed-effects estimator.

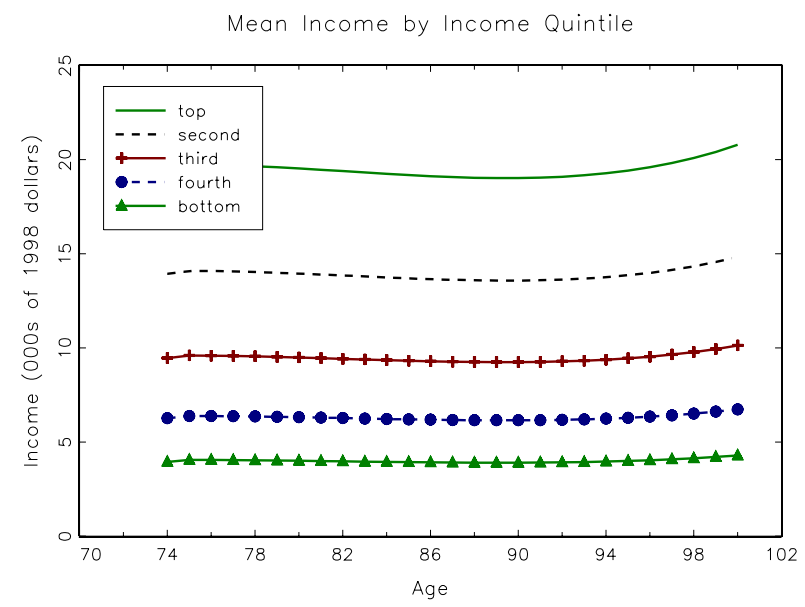

Figure 1: Average income, by permanent income quintile.

Figure 1 presents average non-asset income profiles, conditional on permanent income quintile, computed by simulating our model. In this simulation we do not let people die, and we simulate each person's financial and medical history up through the oldest surviving age allowed in the model. Since we rule out attrition, this picture shows how income evolves over time for the same sample of elderly people. The graph with attrition, however, looks very similar. For those in the top permanent income quintile, annual income averages $\$ 20,000$ per year. Figure 4 below shows that median wealth for the youngest cohort in this income group is slightly under $\$ 200,000$, or about 10 years worth of income. 


\subsection{Mortality and health status}

We estimate the probability of death and bad health as logistic functions of a cubic in age; sex; sex interacted with age; previous health status; health status interacted with age; a quadratic in permanent income rank; and permanent income rank interacted with age.

Using the estimated health transitions, survival probabilities, and the initial joint distribution of age, health, permanent income, and sex found in our AHEAD data, we simulate demographic histories, beginning at age 70. We find that rich people, women, and healthy people live much longer than their poor, male, and sick counterparts. Two extremes illustrate this point: an unhealthy male at the bottom quintile of the permanent income distribution expects to live only 6 more years, that is, to age 76 . In contrast, a healthy woman at the top quintile of the permanent income distribution expects to live 17 more years, thus making it to age 87. Such significant differences in life expectancy should, all else being equal, lead to significant differences in saving behavior. In complementary work (De Nardi et al. [5]), we show this is in fact the case.

We also find that for rich people, the probability of living to very old ages, and thus facing very high medical expenses, is significant. For example, we find that a healthy 70-year-old woman in the top quintile of the permanent income distribution faces a $14 \%$ chance of living 25 years, to age 95 .

\subsection{Co-pay rates}

We calculate the co-insurance rate, $q\left(t, h_{t}\right)$, as the amount spent out of pocket (less insurance premia) divided by total billable medical expenses. Following Yogo [19], we regress the log of this on an age polynomial and health status, and health status interacted with age.

Figure 2 presents average co-pays by age for people in our youngest cohort. Our estimated co-pay profiles are lower for people in bad health and display a non-linear pattern in age. On average, the co-pays display a drop (from $25 \%$ to less than $18 \%$ ) between ages 74 and 87 , but then rise again to about

$19 \%$ after age 87 . This is likely capturing a shift in the composition of medical goods and services that people consume. The rise at the end of life, in particular, could be due to an increase in the fraction of people who, as they age and become more fragile, enter expensive nursing homes that they pay for out of pocket. 


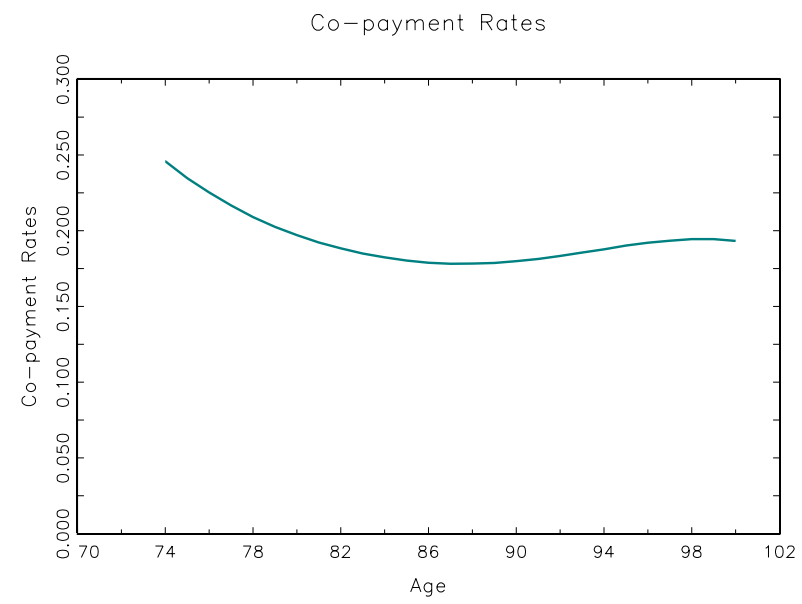

Figure 2: Co-pay rates by age.

\subsection{Medicaid utilization rates}

Although the AHEAD does not provide data on Medicaid expenditures, respondents are asked whether they are currently covered by Medicaid. To interpret these data, we estimate the probability of Medicaid receipt as a function of age, permanent income, health and gender. Figure 3 shows Medicaid participation rates for healthy women at different permanent income percentiles. Figure 3 reveals that at the 10 th income percentile, the Medicaid utilization rate is about $50 \%$ at all ages. For the remaining, higher percentiles Medicaid utilization rises with age. The age trend is most pronounced at the 70th and 90th percentiles, where participation rises from almost zero at age 70 to around $10 \%$ at age 100 .

\subsection{Results}

\subsubsection{Parameter values}

Our estimate of $\beta$, the discount factor is $0.99{ }^{4}$ The estimate of $\nu$, the coefficient of relative risk aversion for "regular" consumption, is 2.15, while the estimate of $\omega$, the coefficient of relative risk aversion for medical goods, is 3.19; the demand for medical goods is less elastic than the demand for consumption. The utility floor is the utility level that one gets when the

\footnotetext{
${ }^{4}$ Standard errors for these estimates can be found in De Nardi et al. [6].
} 


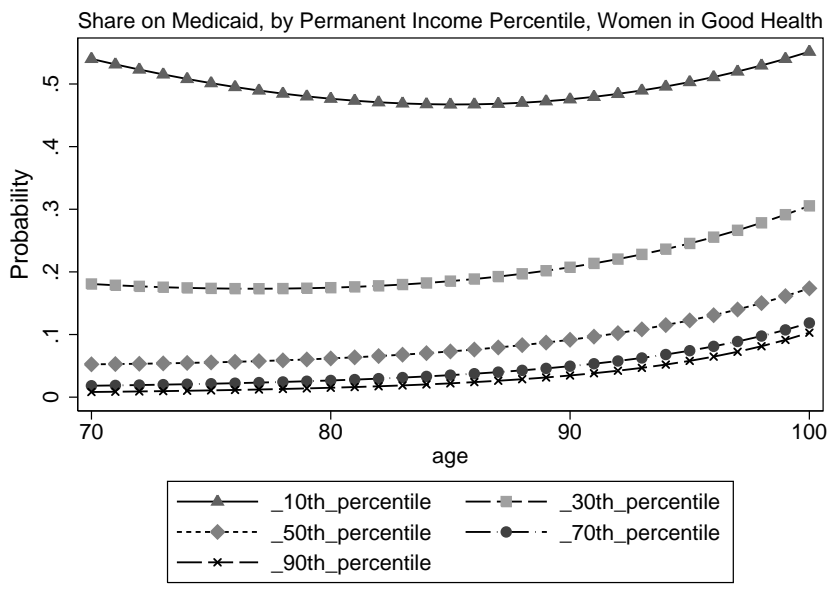

Figure 3: Fraction of healthy women receiving Medicaid, by PI percentile.

medical needs shifter $\mu$ equals 1 and an individual consumes 202 dollars apiece of consumption and medical goods.

We also estimate the coefficients for the mean of the logged medical needs shifter $\mu\left(h_{t}, \psi_{t}, t\right)$, the volatility scaler $\sigma\left(h_{t}, t\right)$ and the process for the shocks $\zeta_{t}$ and $\xi_{t}$. The estimates for these parameters (available from the authors on request) imply that the demand for medical services rises rapidly with age.

We now turn to discussing how well the model fits the net worth and medical expense data and the model's implications for means-tested transfers and total medical expenditures.

\subsubsection{Net worth profiles}

Figure 4 compares the net worth profiles generated by the model (dashed line) and those in the data (solid line) for the members of two birth-year cohorts. It plots median net worth by age and income quintile for those individuals that are still alive at each moment in time, that is, for an unbalanced panel. The lines at the far left of the graph are for the youngest cohort, whose members in 1996 were aged 72-76, with an average age of 74 . The second set of lines are for the cohort aged 82-86 in 1996.

There are five lines for each cohort because we have split the data into permanent income quintiles. However, the fifth, bottom line is hard to distinguish from the horizontal axis because households in the lowest permanent 


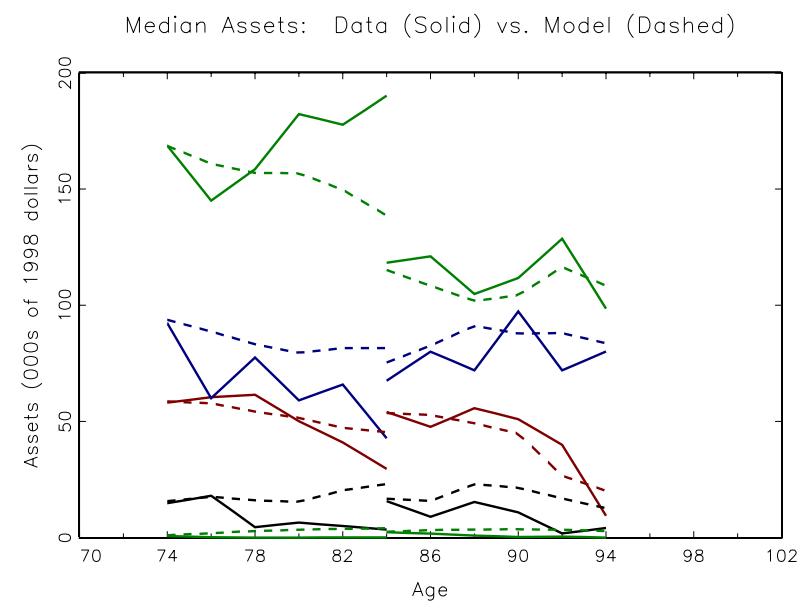

Figure 4: Median net worth by cohort and PI quintile: data (solid lines) and model (dashed lines).

income quintile hold little net worth.

The members of the first cohort appear in our sample at an average age of 74 in 1996. We then observe them in 1998, when they are on average 76 years old, and then again every two years until 2006. The other cohorts start from older initial ages and are followed for ten years, until 2006. The graph reports median net worth for each cohort and permanent-income grouping for six data points over time.

Unsurprisingly, net worth is monotonically increasing in income, so that the bottom line shows median net worth in the lowest income quintile, while the top line shows median net worth in the top quintile. For example, the top left line shows that for the top PI quintile of the cohort aged 74 in 1996, median net worth started at $\$ 170,000$ and then stayed fairly stable over time: $\$ 150,000$ at age $76, \$ 160,000$ at age $78, \$ 180,000$ at ages 80 and 82 , and $\$ 190,000$ at age 84 .

For all permanent income quintiles in these cohorts, the net worth of surviving individuals neither rise rapidly nor decline rapidly with age. If anything, those with high income tend to have increases in their net worth, whereas those with low income tend to have declines in net worth as they age. The profiles for other cohorts, which are shown in our previous paper, are similar. For the most part, the model replicates the main patterns found in the asset data: the most notable exception is that the model overstates 
asset holdings in the second-lowest permanent income quintile.

\subsubsection{Medical expenses}

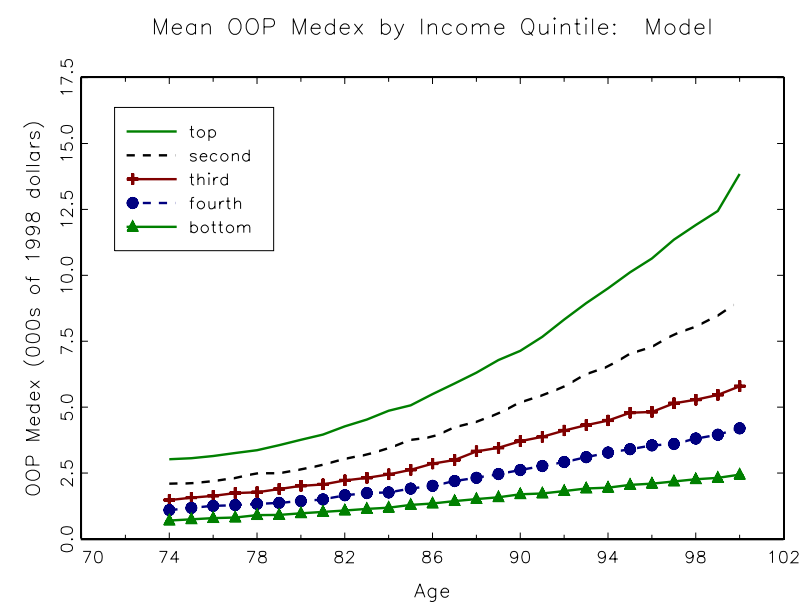

Figure 5: Average out-of-pocket medical expenses by age and permanent income.

Figure 5 displays average out-of-pocket medical expenses (that is, net of Medicaid payments and private and public insurance co-pays) paid by people in the model. Permanent income has a large effect on out-of-pocket medical expenses, especially at older ages. Average medical expenses are less than $\$ 3,000$ a year at age 75 and vary little with income. By age 100 , they rise to $\$ 2,400$ for those in the bottom quintile of the income distribution and over $\$ 14,000$ for those at the top of the income distribution.

Figure 6 compares the out-of-pocket medical expenses generated by the model to those found in the data. The current parameterization of the model underestimates out-of-pocket medical risk at very old ages. As an example, average out-of-pocket medical expenses for the oldest and richest people peak at over $\$ 30,000$, while the model generates just $\$ 16,000$. 


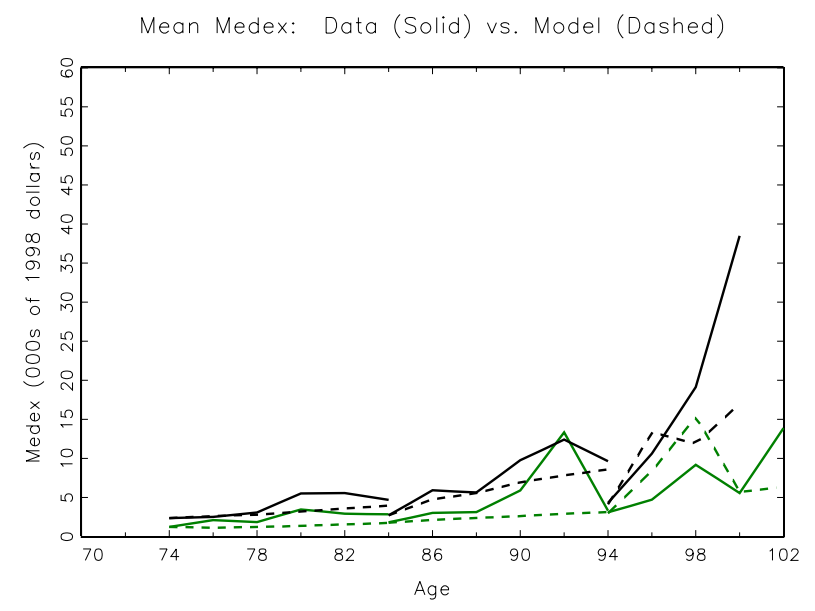

Figure 6: Mean out-of-pocket medical expenses: data (solid lines) and model (dashed lines).

a

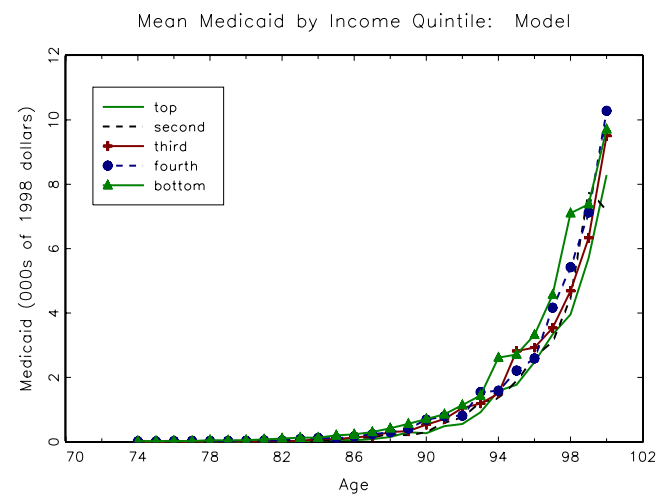

b

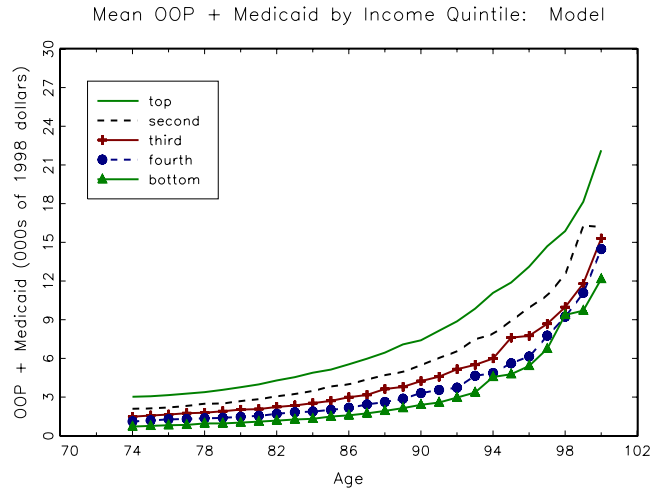

Figure 7: Average medical expenses by age and permanent income. Panel a: paid by Medicaid. Panel b: paid by Medicaid or out-of-pocket. 
Panel a of Figure 7 displays the average medical expenses covered by our means-tested social insurance program, measured as the increase in $q_{t} m_{t}$ generated by government transfers. "Medicaid" payments rapidly increase with age, going from roughly zero at age 74 to nearly $\$ 9,000$ at age 100 . Consistent with the redistributive nature of the program, these payments are quite close across people of different permanent incomes, but are higher for the poor. Panel b of Figure 7 shows the sum of medical expenses paid out-of-pocket and the expenses paid by Medicaid. Medicaid allows poorer people to consume proportionally much more medical goods and services than they pay for. As a result, the expense sum shown in panel $b$ rises more slowly with income than the out-of-pocket expenditures shown in Figure 5. At age 100, people in the top permanent income quintile spend $470 \%$ more out-of-pocket than people in the bottom quintile. Once Medicaid is included, the difference narrows to $80 \%$.

a

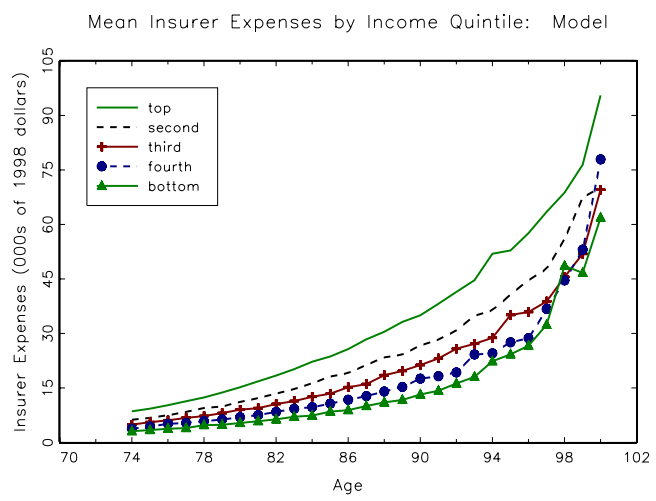

$\mathrm{b}$

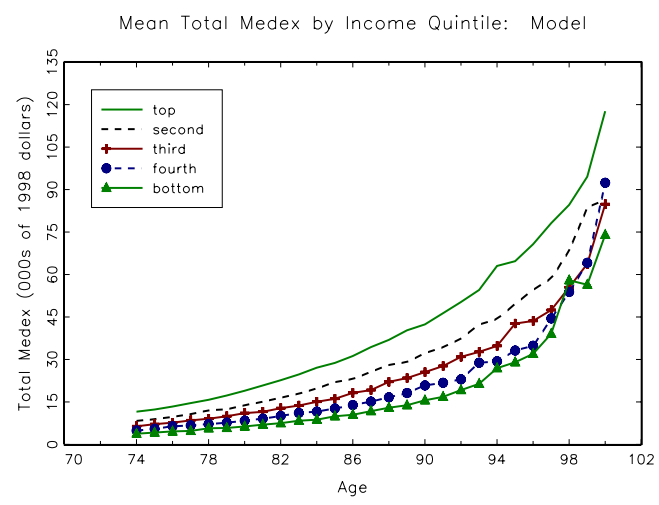

Figure 8: Average medical expenses by age and permanent income. Panel a: paid by insurers. Panel b: total.

Panel a of Figure 8 displays average medical expenses covered by private and public insurers. These payments are very large and also increase by age and permanent income, reaching over $\$ 90,000$ for the oldest members of the top permanent income quintile. The oldest in the poorest permanent income quintile, however, also benefit from these payments, which reach over $\$ 60,000$ at age 100. Panel b of Figure 8 displays total medical expenses, which in this case also coincides with total consumption of medical goods and services. Comparing the two panels makes it clear that most elderly individuals consume far more medical care than they for pay out-of-pocket. 
The increase in total medical expenses after retirement is very large, going from around $\$ 10,000$ at age 74 to $\$ 60$ to $\$ 100$ thousand at age 99 .

\subsection{Utility floor, preference shocks, and implied insur- ance system}

Through the interaction of the utility floor and medical needs shocks, the model has interesting implications on the insurance provided by meanstested programs. Our utility floor is based on the consumption of $\$ 202$ in medical goods and $\$ 202$ of non-medical goods with the medical preference shifter equalling 1 . The interpretation of this number is not obvious, however, because people with higher medical needs receive larger transfers.

a

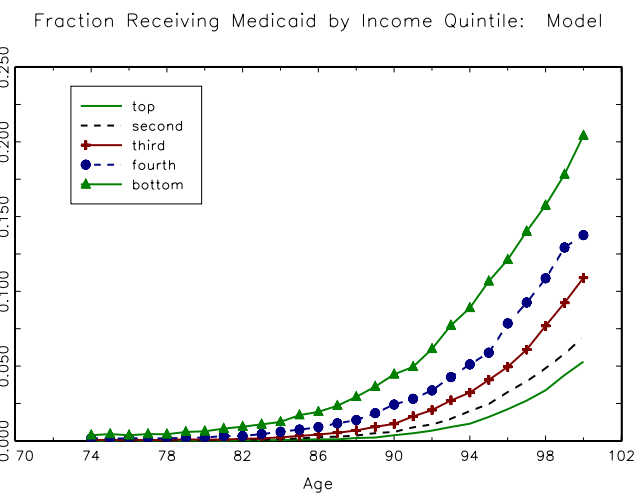

$\mathrm{b}$

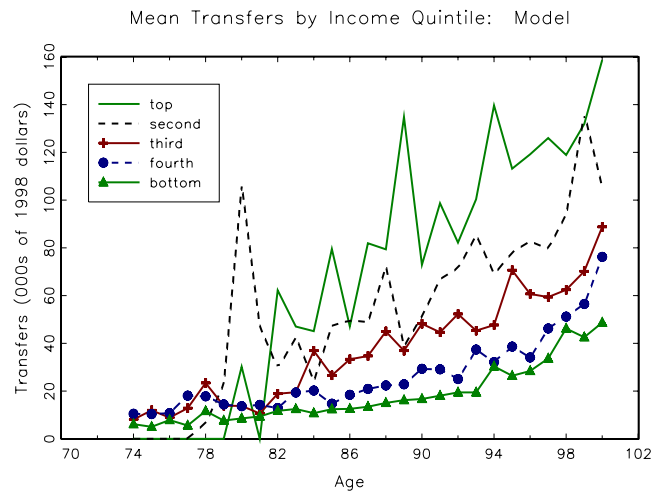

Figure 9: Means-tested transfers. Panel a: fraction receiving transfers. Panel b: average transfers per recipient.

Figure 9 describes the transfers generated by the model. Panel a of this figure shows the fraction of individuals receiving transfers, while panel $b$ shows transfers per recipient. Although the model implies lower Medicaid participation rates than found in the data, it captures the way in which Medicaid usage increases with age. Initially, very few people receive transfers, but as people age, and medical needs increase, more people become eligible. By age 100, over $10 \%$ of people receive transfers. The vast majority of the transfers are received by people with large medical needs and are thus spent on medical goods and services, rather than on non-medical consumption. Because people in the top permanent income quintile receive transfers only 
when their medical needs are extremely severe, very few of them receive transfers, but the average transfer is high. Even after age 95, only about $4 \%$ of this group receive transfers, with an average transfer in excess of $\$ 100,000$. In contrast, after age 95 , the average transfer in the bottom quintile is less than $\$ 50,000$, but over $16 \%$ of this group receive transfers. Because the distribution of the medical needs shifter $\mu_{t}$ does not depend directly on income, the increased rate of recipiency found in the bottom income quintile means that the poor on average receive more transfers than the rich; see the discussion of Figure 7.

At any income level, however, the baseline parameterization of the consumption floor, coupled with our estimated medical needs shocks, can lead to very large transfers, which provide significant insurance against devastating medical illnesses.

\section{$6 \quad$ Policy experiments}

In the spirit of the recently debated health reforms, we study the effects of making public health insurance more generous. In the first experiment, we analyze an increase in the generosity of Medicaid by raising the utility floor by $50 \%$. In the second experiment, we analyze an increase in the number of insured individuals by reducing co-payment rates by $25 \%$.

\subsection{A more generous means-tested program}

In this policy experiment, we increase the generosity of means-tested insurance by increasing the level of the utility floor by $50 \% .^{5}$ Figure 10 shows how transfers vary with the preference shifter $\mu_{t}$ for both the benchmark and the experiment with the higher utility floor. Figure 10 shows that in order to maintain a higher utility floor, transfers become much larger at all levels of medical need.

This increase in the insurance provided by the government leads people to save less for medical needs, and generates large reductions in net worth. Panel a of Figure 11 plots the net worth of the youngest cohort for the benchmark calibration (dashed line) and for the experiment with more generous means-tested programs (solid line). Households deplete their assets more

\footnotetext{
${ }^{5}$ More precisely, we increase the consumption equivalent $\underline{c}$ by $50 \%$.
} 


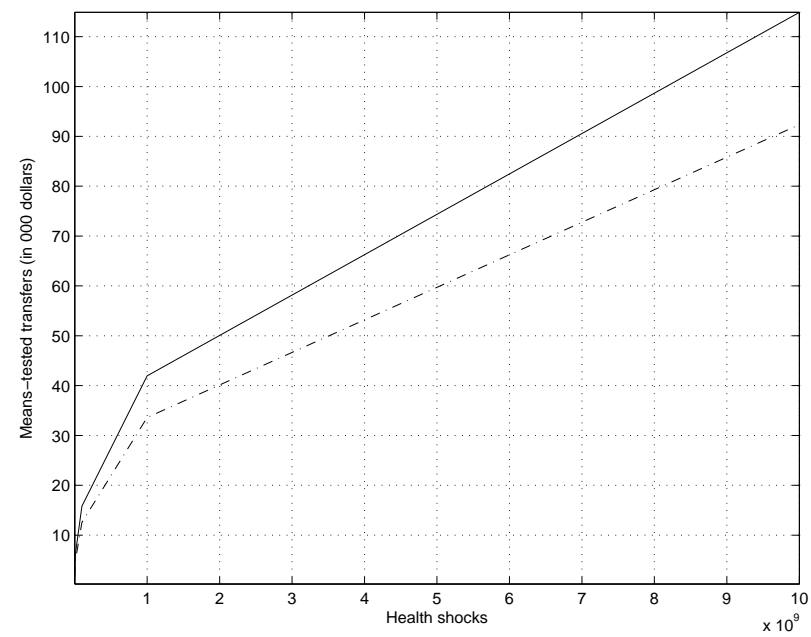

Figure 10: Means-tested transfers as a function of $\mu(\cdot)$. Dashed line: benchmark, solid line: experiment with more generous utility floor.

quickly in the specification with more generous insurance. The median asset holding of 95-year-old people in the highest permanent income quintile drops $22 \%$, from $\$ 57,000$ to $\$ 44,000$; the median asset holding of 95-yearold people in the lowest permanent income quintile drops $51 \%$, from $\$ 1,900$ to $\$ 900$. The declines are thus proportionally much larger for poorer people, who are the ones most likely to benefit to benefit from a means-tested transfer program. However, richer people also risk being wiped out by large medical expenses and thus benefit from the increased insurance provided by the higher consumption floor.

Raising the utility floor affects medical expenditures in several ways. The obvious direct effect of a higher floor is to raise the medical expenditures of individuals eligible for assistance. Moreover, raising the floor reduces the need to save, which will, holding assets fixed, lead individuals to increase their consumption of both medical and non-medical goods. Reduced saving, on the other hand, will lower medical expenditures in the future. Panel b of Figure 11 shows that a more generous utility floor increases total consumption of medical goods and services, especially after age 90 and for those in the bottom two permanent income quintiles. 
a

Median Assets: Experiment (Solid) vs. Baseline (Dashed)

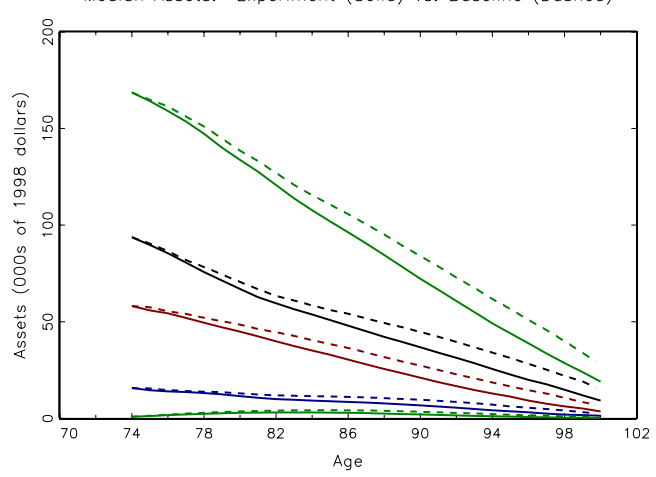

$\mathrm{b}$

Mean Total Medex: Experiment (Solid) vs. Baseline (Dashed)

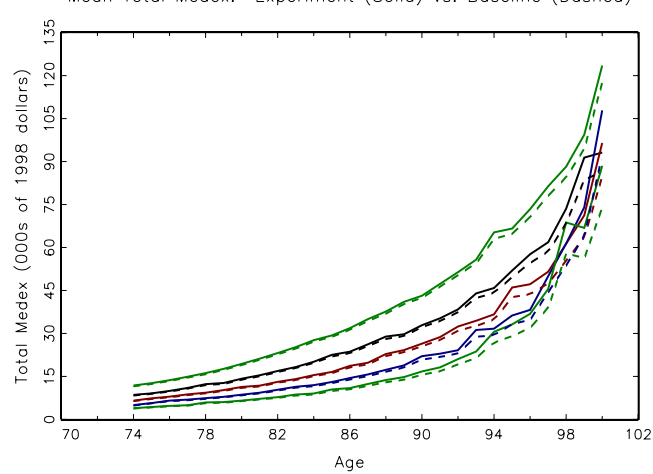

Figure 11: Net worth (panel a) and total medical expenses (panel b) by age and permanent income. Dashed line: benchmark, solid line: experiment with more generous utility floor.

a

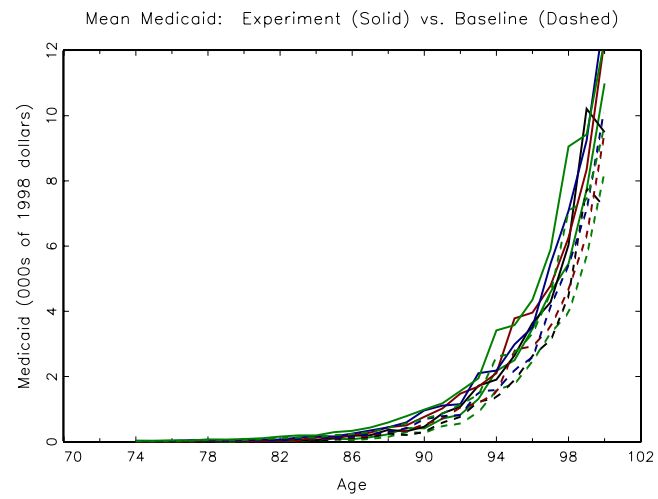

$\mathrm{b}$

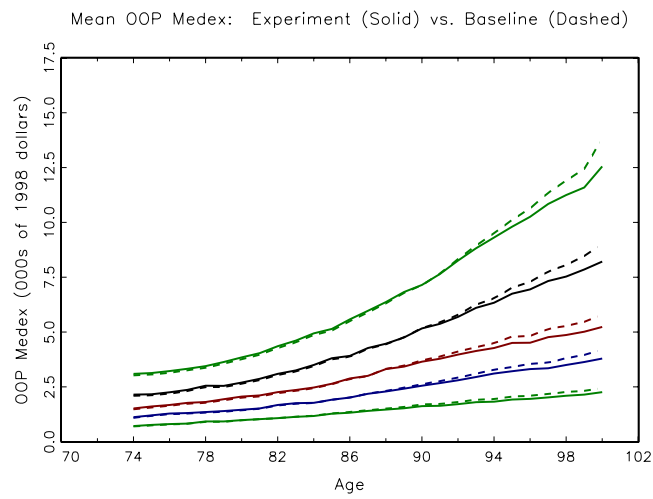

Figure 12: Medicaid payments (panel a) and out-of-pocket medical expenses (panel b) by age and permanent income. Dashed line: benchmark, solid line: experiment with more generous utility floor. 
Panel a of Figure 12 shows that the higher utility floor, along with the resulting decrease in assets, increases Medicaid payments. Panel b shows that a more generous insurance system reduces out-of-pocket medical expenditures; the reduction in the consumers' cost share outweighs the increase in total medical expenditures.

\subsection{A more generous co-insurance system}

In this policy experiment we reduce the co-payment schedule by $25 \%$. As in the previous experiment, the households react to the increased insurance by running down their assets more rapidly. This experiment, however, has smaller effects than the previous one (see Figure 13), and the largest effects occur at earlier ages. For example, the assets of 95-year-old people in the top permanent income group drop by $12 \%$, while the assets in the bottom group change by $0.1 \%$.

a

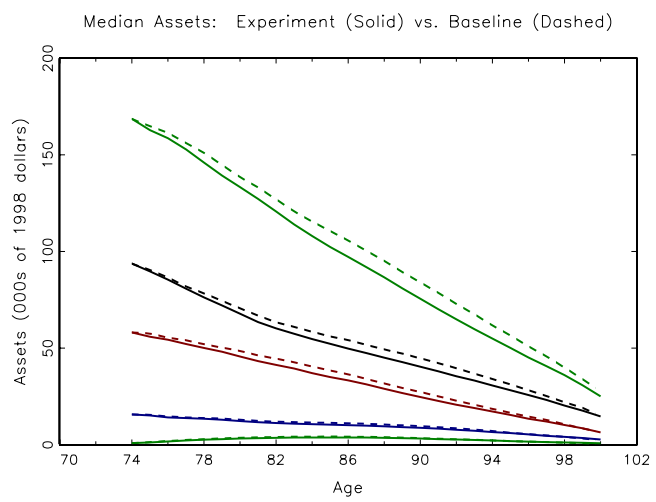

b

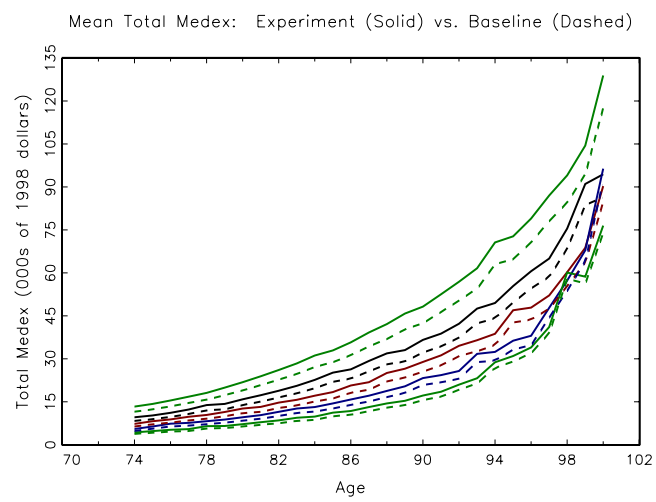

Figure 13: Net worth (panel a) and total medical expenses (panel b). Dashed: benchmark, solid: more generous co-insurance system.

Panel b of Figure 13 shows that total medical expenses go up at all ages, especially for households in the highest permanent income quintiles. While the largest increases in absolute terms occur at the oldest ages, the increase at younger ages represent larger proportions.

Figure 14 shows that reducing the co-pay rates reduces out-of-pocket medical costs at all ages, especially for those with higher permanent income. Because individuals in the lower income quintiles rely more heavily on Medicaid, they are less likely to incur co-pays. As a result, their medical care 
decisions are less sensitive to changes in co-payment rates. While lower copay rates increase total medical expenditures, this increase in quantities is more than offset by the reduction in the consumers' out-of-pocket shares. Medicaid payments go down for similar reasons. The increase in total medical expenses is thus borne entirely by insurers, as shown in Figure 15. Given that Medicare is by far the principal insurer for retirees, we see an important interaction between the Medicaid and Medicare programs: increases in the generosity of Medicare reduce Medicaid payments.

Our finding that a decrease in the out-of-pocket price of medical expenditures leads to a reduction in out-of-pocket expenditures indicates that the elasticity for medical goods is fairly small. In a recent study, Fonseca et al. [10] calculate that the co-insurance elasticity for total medical expenditures ranges from -0.27 to -0.35 , which they find to be consistent with existing micro evidence. Repeating their experiment (a $150 \%$ increase in copay rates) with our model reveals that elasticities range widely by age and income: richer and younger people have higher elasticities. To calculate a summary number, we use our model of mortality and an annual population growth rate of $1.5 \%$ to find a cross-sectional distribution of ages. Combining this number with our simulations, we find an aggregate cross-sectional elasticity of -0.46 .

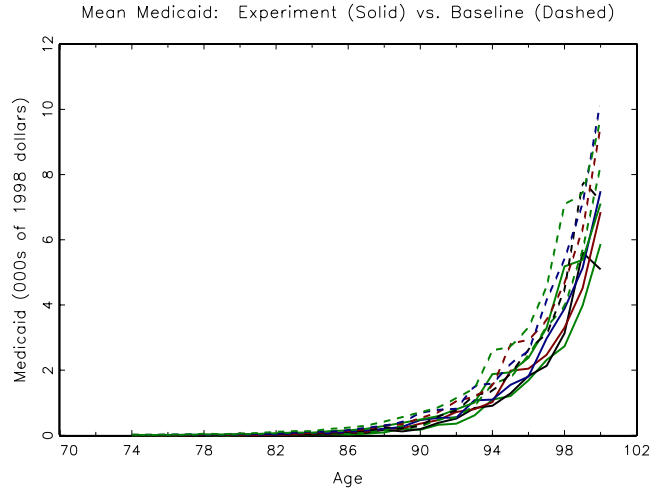

$\mathrm{b}$

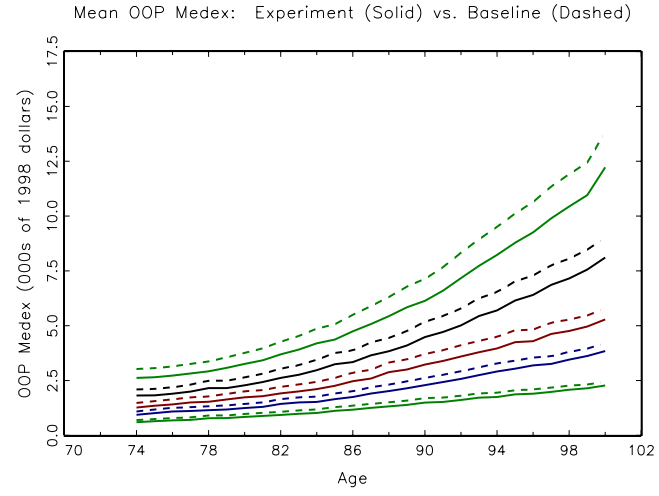

Figure 14: Medicaid payments (panel a) and out-of-pocket medical expenses (panel b) by age and permanent income. Dashed line: benchmark, solid line: experiment with more generous co-insurance system. 


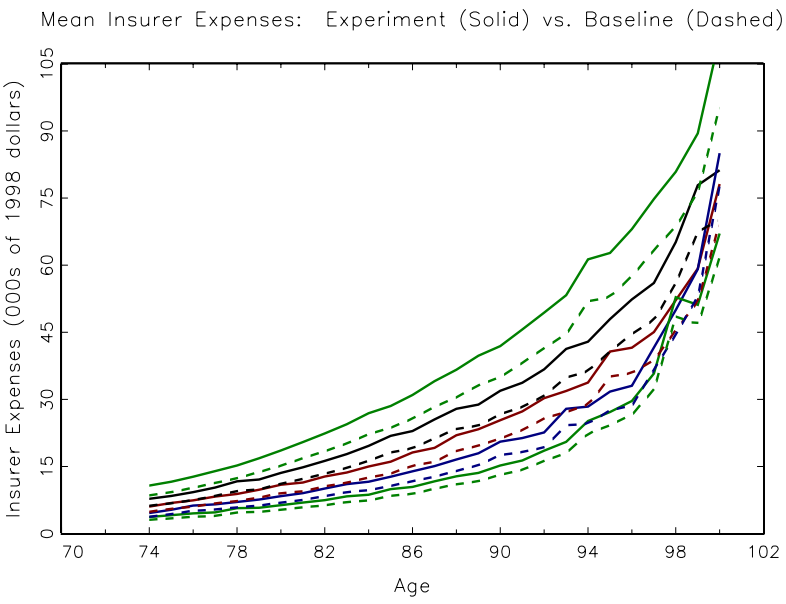

Figure 15: Medical insurer payments by age and permanent income. Dashed line: benchmark, solid line: experiment with more generous co-insurance system.

\section{Conclusions and extensions}

In this paper we use a model of saving and endogenous medical expenditure to study reforms to the Medicare and Medicaid systems. We find that the insurance provided by Medicaid is important at all income levels. Although richer individuals use Medicare much less frequently than poorer ones, the transfers they do receive often cover catastrophic medical expenditures. As a result, making Medicaid more generous reduces saving in all income groups.

We also consider the effects of reducing the price of medical care, by reducing co-payment rates. We find that the demand for medical services is fairly inelastic (less than one-half), so that decreases in co-pays lead to decreases in out-of-pocket medical expenses. This low elasticity is consistent with estimates from several other studies. Because individuals in the lower income quintiles rely more heavily on Medicaid, their medical care decisions are less sensitive to changes in co-pays. Reducing co-payment rates has a larger effect on the out-of-pocket medical expenditures of the rich.

We finish by considering a few promising extensions. Our model suggests that changes in the Medicaid program lead to large changes in saving. This 
in turn suggests that the insurance provided by Medicaid has a large welfare effects. One way to test such a proposition is to measure the value of Medicaid through compensating differential calculations like those in Brown and Finkelstein [2]. These benefits can then be compared to Medicaid expenditures.

The model used in this paper uses parameter values taken from De Nardi et al. [6]. While the model does a good job of matching other features of the data, it understates Medicaid participation. One way to improve the model's fit along this dimension is to expand our MSM criterion function to include Medicaid participation rates and re-estimate the model's parameters. Preliminary estimates using this approach show that making the utility floor more generous significantly increases simulated Medicaid participation. Continued work in this direction should lead to a more realistic model. 


\section{References}

[1] Robert Brook, John E. Ware, William H. Rogers, Emmett B. Keeler, Allyson R. Davies, Cathy A. Donald, George A. Goldberg, Kathleen N. Lohr, Patricia C. Masthay, and Joseph P. Newhouse. Does free care improve adults' health? results from a randomized trial. New England Journal of Medicine, 309(23):1426-1434, 1983.

[2] Jeffrey Brown and Amy Finkelstein. The interaction of public and private insurance: Medicaid and the long term care insurance market. American Economic Review, 98(3):1083-1102, 2008.

[3] David Card, Carlos Dobkin, and Nicole Maestas. Does medicare save lives? NBER Working Paper 13668, 2007.

[4] Joseph J. Jr. Doyle. Returns to local-area healthcare spending: Using health shocks to patients far from home. Mimeo, 2010.

[5] Mariacristina De Nardi, Eric French, and John B. Jones. Life expectancy and old age savings. American Economic Review: Papers and Proceedings, 99(2):110-115, 2009.

[6] Mariacristina De Nardi, Eric French, and John B. Jones. Why do the elderly save? the role of medical expenses. Journal of Political Economy, 118(1):39-75, 2010.

[7] Zhigang Feng. Macroeconomic consequences of alternative reforms to the health insurance system in the u.s. Mimeo, 2009.

[8] Amy Finkelstein and Robin McKnight. What did medicare do (and was it worth it)? NBER Working Paper 11609, 2005.

[9] Elliott S. Fisher, David E. Wennberg, Therese A. Stukel, Daniel J. Gottlieb, F.L. Lucas, and Etoile L. Pinder. The implications of regional variations in medicare spending. part 2: Health outcomes and satisfaction with care. Annals of Internal Medicine, 138(4):288-322, 2003.

[10] Raquel Fonseca, Pierre-Carl Michaud, Titus Galama, and Arie Kapteyn. On the rise of health spending and longevity. Rand Working Paper WR$722,2009$. 
[11] Eric French and John Bailey Jones. On the distribution and dynamics of health care costs. Journal of Applied Econometrics, 19(4):705-721, 2004.

[12] Michael Grossman. On the concept of health capital and the demand for health. Journal of Political Economy, 80(2):223-255, 1972.

[13] R. Glenn Hubbard, Jonathan Skinner, and Stephen P. Zeldes. Precautionary saving and social insurance. Journal of Political Economy, 103(2):360-399, 1995.

[14] Ahmed Khwaja. Estimating willingness to pay for medicare using a dynamic life-cycle model of demand for health insurance. Journal of Econometrics, forthcoming, 2009.

[15] Karen Kopecky and Tatyana Koreshkova. The impact of medical and nursing home expenses and social insurance policies on savings and inequality. Mimeo, 2009.

[16] The Kaiser Commission on Medicaid and the Uninsured. Medicaid: A Primer. Menlo Park, CA, The Henry J. Kaiser Family Foundation, 2010.

[17] Susann Rohwedder, Steven J. Haider, and Michael Hurd. Increases in wealth among the elderly in the early 1990s: How much is due to survey design? Working Paper 10862, National Bureau of Economic Research, 2004 .

[18] John Karl Scholz, Ananth Seshadri, and Surachai Khitatrakun. Are americans saving optimally for retirement? Journal of Political Economy, 114:607-643, 2006.

[19] Motohiro Yogo. Portfolio choice in retirement: Health risk and the demand for annuities, housing, and risky assets. Mimeo, University of Pennsylvania, 2009. 